EPJ Web of Conferences 31, 00030 (2012)

DOI: $10.1051 /$ epjconf/20123100030

(C) Owned by the authors, published by EDP Sciences - SIF, 2012

\title{
Towards ion identification limit with the silicon telescopes of the FAZIA project
}

\author{
S. CARBONI \\ for the FAZIA COLLABORATion
}

INFN, Sezione di Firenze, Italy

\begin{abstract}
The response of Si-Si-CsI(Tl) and $\mathrm{Si}-\mathrm{CsI}(\mathrm{Tl})$ telescopes with quality controlled detectors, developed within the FAZIA collaboration, is presented. The aim of the test experiment was to explore the capabilities of various solutions exploiting digital pulse shape analysis techniques for charge and mass separation of ions stopped in a single silicon layer, in order to decrease the identification threshold. It has been found that it is possible to fully discriminate the charge of stopped ions up to the maximum available $\mathrm{Z}$ (slightly over that of the beam, $\mathrm{Z}=54)$. The $\Delta \mathrm{E}-\mathrm{E}$ correlation, both from the first two silicon detectors and for $\mathrm{Si}+\mathrm{Si}$ vs. $\mathrm{CsI}(\mathrm{Tl})$, can identify all the isotopes up to $\mathrm{Z}=25$. The radiation damage effect on these FAZIA silicon detectors has been also studied. In particular, some results about the effect due to the punching-through and stopped Xe ions will be shown and discussed.
\end{abstract}

A $35 \mathrm{MeV} /$ nucleon ${ }^{129} \mathrm{Xe}$ beam, delivered by the "Ciclotrone Superconduttore" (CS) of the "Laboratori Nazionali del Sud" (LNS) in Catania, was used for bombarding thin targets of ${ }^{n a t} \mathrm{Ni}$ and $\mathrm{Au}$ to test the capabilities of the FAZIA telescope prototypes.

Seven different telescopes were mounted in the "Ciclope" scattering chamber at LNS, in the vicinity of the grazing angles for the investigated reactions, in order to have the largest variety of outgoing reaction products. The standard FAZIA telescope analyzed here consisted of three elements: a first $(300 \mu \mathrm{m})$ silicon, a second $(300 \mu \mathrm{m})$ silicon and a $\approx 4 \mathrm{~cm}$ thick $\mathrm{CsI}(\mathrm{Tl})$ permits unrestricted use, distribution, and reproduction in any medium, provided the original work is properly cited. 
scintillator with photodiode $(\mathrm{PhD})$ readout. In the following, the first and the second silicon detector of a three-element telescope will be addressed as Si1 and Si2, respectively.

Finally, a single $300 \mu \mathrm{m}$ silicon detector (motorized so that it could slide to angles $\sim 0.5^{\circ}$ very close to the beam direction) was devoted at studying the effects of radiation damage (Tele RD). For major details on the experiment setup see [1].

All the silicon detectors are ion-implanted, of the nTD type, with bulk resistivity values in the range $3000-4000 \Omega \mathrm{cm}$. They are all in the so-called "transmission mounting", with thin dead layers on both sides. The $300 \mu \mathrm{m}$ thick detectors were manufactured by FBK (Trento, Italy).

The improvement in the identification capabilities observed in this experiment is the result of several "ingredients", as it was learnt during the R\&D preparatory phase $[1,2]$ :

1. "random" cut of the silicon wafers. This reduce the effects related to the crystal orientation [3].

2. usage of silicon detectors with good dopant homogeneity. Only detectors with doping inhomogeneities of about $1 \%$ or better were used.

3. selection of silicon detectors with good planarity and parallelism of front and rear sides (flatness).

4. reverse mounting configuration of silicon detectors [4-7].

5. careful control of the constancy of the electric field profile in the detectors.

6. usage of dedicated preamplifiers, located inside the vacuum chamber as close as possible to the respective detectors.

7. extensive optimization of the digital treatment of the sampled pulseshapes of both the charge and current signals.

Energy measurements, which are needed for all the identification techniques, are obtained from the charge signals by means of digital trapezoidal shaping. The parameters of trapezoidal filter have been adjusted to reduce to less than $1 \%$ the ballistic deficit.

From the obtained Si1-Si2 energy correlation (see Table 1) one sees that all produced elements (up to $\mathrm{Z}=54$ and slightly above) are clearly identified over the whole dynamic range. 
In order to perform a quantitative estimation of the obtained identification, a standard linearization procedure was applied to the $\Delta \mathrm{E}-\mathrm{E}$ correlation. The so-called Particle Identification (PID) parameter was then obtained with a linear interpolation between adjacent Z-lines [2].

With reference to the PID spectrum, the isotopic discrimination limit can be quantitatively evaluated by means of a "Figure of Merit" (FoM), introduced in [9], and defined as:

$$
F o M=\frac{\left|\overline{P I D_{2}}-\overline{P I D_{1}}\right|}{F W H M_{1}+F W H M_{2}}
$$

where $\overline{P I D_{1}}$ and $\overline{P I D_{2}}$ are the centroids of the peaks associated to two neighboring isotopes and $F W H M_{1}$ and $F W H M_{2}$ are their full widths at half maximum.

We conventionally consider two isotopes as "well separated" if their figure of merit is greater than 0.7 (see [2]), which corresponds to a peak-to-valley ratio of 2.0 when the two peaks have the same integral. FoM greater than 0.7 up to $Z \simeq 21-22$ are observed and this represents, according to our convention, the limit for good isotopic discrimination. Isotopes appear to be reasonably separated also for heavier elements, up to $Z \sim 25$, where the calculated FoM is $\sim 0.5$.

One may wonder whether our identification capability has arrived close to the physical limit imposed by energy straggling. We have chosen to run simulations using just two simple recipes. The first one is the pure Bohr straggling which is some kind of standard reference, although it is expected to give a lower limit for the effect in our energy domain. The second one is a semi-empirical parametrization by Yang [10], based on experimental data for heavy ions up to $\mathrm{Z}=18$ (i.e. reasonably appropriate for a comparison with our data). We have produced $\Delta \mathrm{E}-\mathrm{E}$ correlations as the one obtained experimentally and FoM between neighbouring ions has been calculated.

Table 1: Characteristics and placements of the telescopes used in the experiment at LNS.

\begin{tabular}{ccccc}
\hline Telescope & Silicon 1 & Silicon 2 & CsI(Tl) readout & Position \\
\hline Tele D & $300 \mu \mathrm{m}$ & $300 \mu \mathrm{m}$ & $3.6 \mathrm{~cm}+\mathrm{PhD}$ & $2.6^{\circ} \pm 0.1^{\circ}$ \\
Tele RD & $300 \mu \mathrm{m}$ & - & - & $\simeq 0.5^{\circ}$ moving \\
\hline
\end{tabular}




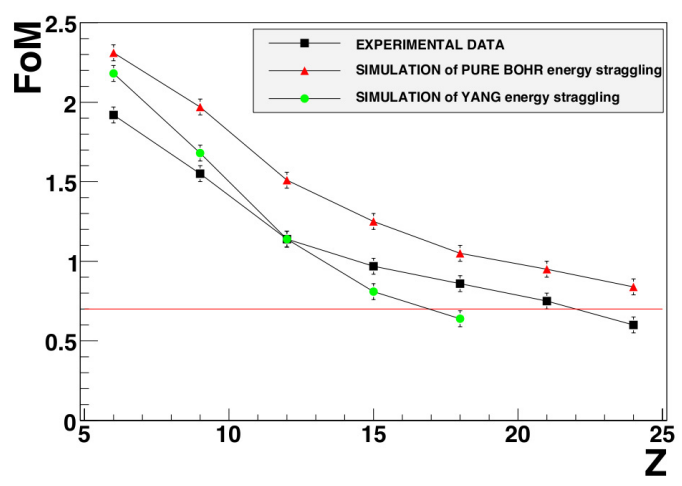

Figure 1: Figure of Merit (FoM) calculated for pairs of adjacent isotopes of different $\mathrm{Z}$ for the experimental data (black squares) and for two Monte Carlo simulations, one with pure Bohr straggling (red triangles) and one with the parametrization of Yang [10] (green points). The horizontal line shows the level for "good" discrimination (Colour online).

The comparison between simulated and experimental results is shown in Fig. 1. The indicated error bars take into account the non reproducibilities of linearization and FoM calculation, which are larger than the statistical errors. As expected, the Bohr model gives a smaller straggling and hence a better FoM for all the considered elements. Experimental FoM are all below Bohr's values and are closer to Yang's estimates, although the behavior seems somewhat "flatter" as a function of Z. The comparison indicates that we are not very far (maybe within 10-20\%) from the physical limit imposed by the energy straggling phenomena, and hence one might conclude that there is not much room for further improvements of the technique.

Equally good isotopic discrimination has been reached for most penetrating particles passing through both silicon detectors and reaching the $\mathrm{CsI}(\mathrm{Tl})$ scintillator, using the $\Delta \mathrm{E}(\mathrm{Si} 1+\mathrm{Si} 2)-\mathrm{E}(\mathrm{CsI})$ correlation. Elements are fully identified over the whole dynamic range, while isotopic discrimination is present up to $\mathrm{Z} \simeq 26$.

Two methods of PSA have been used for the data presented here and they are based on the two correlations "Energy vs Charge rise-time" and "Energy vs Current maximum". Both techniques had been already investigated within the FAZIA R\&D program. The "rise-time of the charge signal" has 
been defined as the time difference between two digital Constant Fraction Discriminators with cubic interpolation [8]. Two fractions, $20 \%$ and $70 \%$, have been empirically recognized as providing the best performances. The "maximum of the current signal" (Imax) has been estimated, after baseline subtraction, as the absolute maximum of the interpolated current waveform (with cubic interpolation [8]).

In order to have large dynamic ranges (cf Table 1), rather low amplification values had to be used in the present experiment. A consequence of this choice was that no isotopic resolution with PSA could be observed, at variance with other FAZIA experiments using higher amplifications [2,3]. Therefore the identification thresholds and FoM calculated hereafter for PSA refer only to charge discrimination.

We have tested the PSA capabilities on Si2 because the correlation on Si1 had low statistics in the identification threshold zone.

The comparison between the correlations for the two techniques shows that the PSA based on the "Energy vs Current maximum" method gives a better identification than the "Energy vs Charge rise-time". This is especially evident for fragments with $\mathrm{Z}=1-2$ and $\mathrm{Z}=53$.

Fig. 2 shows the measured FoM value as a function of energy for several pairs of elements. The chosen value $(\mathrm{FoM}=0.7)$ for "good" identification is indicated by the horizontal line. One can see that for fragments up to $\mathrm{Z} \simeq 20$, we have FoM $>0.7$ in the whole energy range, while for heavier elements the resolution gets increasingly worse with decreasing energy.

The plot of Fig. 3 shows the sizable lowering of the energy threshold which can be obtained by employing PSA techniques on the first silicon detector (full red points for "Energy vs Charge rise-time" method and open blue squares for "Energy vs Current maximum" method) with respect to the more conventional $\Delta \mathrm{E}-\mathrm{E}$ technique (black triangles). In this second case, the threshold is simply the energy necessary to pass through the whole thickness $(300 \mu \mathrm{m})$ of the first silicon detector. On the left part of Fig. 3, the thresholds are expressed in terms of the total energy, on the right part in terms of energy per nucleon (for heavy nuclei, the mass has been estimated with the formula $\left.A=2.08 Z+0.0029 Z^{2}[11]\right)$.

Now we report on the first test dedicated to the study of "radiation damage and PSA". Here we consider the single $300 \mu \mathrm{m}$ silicon detector (Tele $\mathrm{RD}$ in Table 1) mounted in reverse configuration on a motorized support.

Three areas were defined, called "Implantation damage", "Punching through damage" and "Standard irradiation". Each of them was delimited by a collimator constituted by a circular hole with diameter of $3 \mathrm{~mm}$. In the "Implantation damage" zone, the collimator was covered by a $300 \mu \mathrm{m}$ 


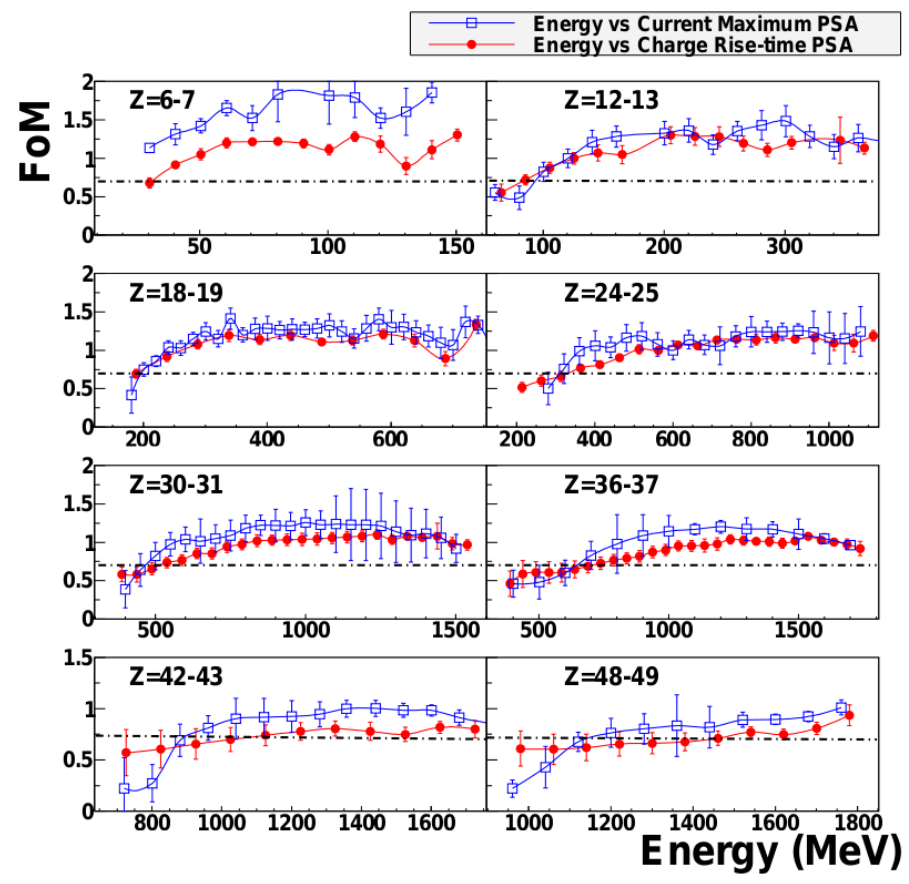

Figure 2: The Figure of Merit as a function of Energy for pairs of adjacent $\mathrm{Z}$ values, identified with different PSA techniques: "Energy vs Charge rise-time" (red points) and "Energy vs Current maximum" (blue squares) (Colour online).

of silicon acting as absorber, while in the other positions no absorbers were put in front of the holes. The absorber was necessary in order to reduce the energy of elastically scattered Xe ions, so that they stop in the detector.

The detector was ciclically irradiated and tested for energy and risetime resolution. Radiation damage effects were mainly studied by controlling the performance in terms of energy and rise time of the signals of the elastically scattered ions. Signals were acquired only in the testing position, while during the irradiation in the high fluence zone, no data were acquired, but a scaler counting pulses from a CFD registered the number of hitting ions as a function of solar time.

It is important to underline that also for this detector the bias voltage is provided by a remotely controlled high voltage power supply, that includes also a current monitoring system and keeps the voltage at detector electrodes constant during the experiment. 

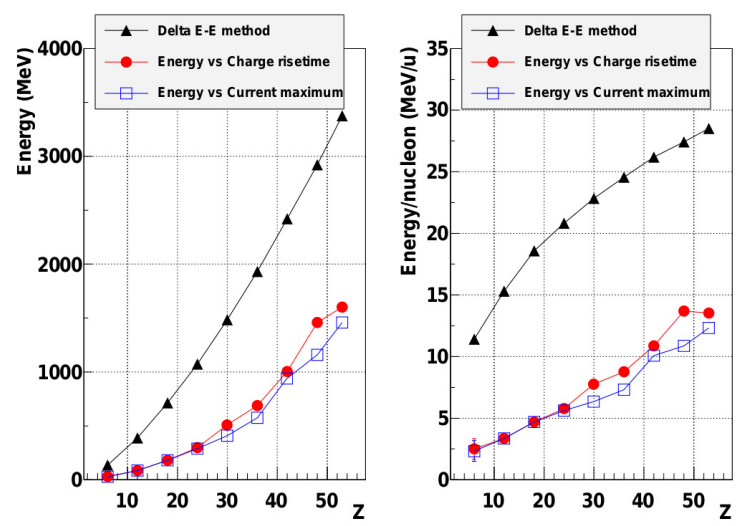

Figure 3: Energy thresholds for $\mathrm{Z}$ identification with $\Delta \mathrm{E}(300 \mu \mathrm{m})$-E technique (black triangles) and with PSA techniques (red points and blue squares) as a function of atomic number Z (Colour online).

Not surprisingly, the "implantation damage" zone showed the strongest variations. At the end of the measurement, after a $6 \cdot 10^{8} / \mathrm{cm}^{2}$ fluence, a $20 \%$ pulse height defect and a $50 \%$ decrease of charge risetime was observed for Xe ions. These values reduce to $1 \%$ and less than $5 \%$ for the punchingthrough zone. The standard irradiation zone (fluence $\leq 2.5 \cdot 10^{6} / \mathrm{cm}^{2}$ as in a real life experiment) featured remarkably constant performances both in charge and timing. This implies that once the truly applied voltage on the detector is kept constant, the irradiated zones damage does not jeopardize the detector performance in undamaged regions.

\section{References}

[1] S. Carboni et al., Nucl. Instr. and Meth. A 664 (2012) 251.

[2] L. Bardelli et al., Nucl. Instr. and Meth. A 654 (2011) 272.

[3] L. Bardelli et al., Nucl. Instr. and Meth. A 605 (2009) 353.

[4] J.B.A. England, G.M. Field, T.R. Ophel, Nucl. Instr. and Meth. A280 (1989), 291.

[5] G. Pausch Nucl. Instr. and Meth. A 337 (1994) 573. 


\section{EPJ Web of Conferences}

[6] M. Mutterer et al., IEEE Trans. Nucl. Science, 47 no.3 (2000) 756.

[7] S. Barlini et al., Nucl. Instr. and Meth. A 600 (2009) 644.

[8] L. Bardelli et al., Nucl. Instr. and Meth. A 521 (2004) 480.

[9] R.A. Winyard, J.E. Lutkin, G.W. McBeth, Nucl. Instr. and Meth. 95 (1971) 141.

[10] Q. Yang, D.J. O’Connor, Nucl. Instr. and Meth. B 61 (1991) 149.

[11] K.X. Jing Nucl. Phys. A 645 (1999) 203. 